\title{
COMPOSIÇÃO E ATIVIDADE DE MOSQUITOS CULICIDAE. EMPREGO DE ARMADILHA CDC NO VALE DO RIBEIRA, ESTADO DE SÃO PAULO, BRASIL
}

Almério de Castro Gomes* Oswaldo Paulo Forattini* Délsio Natal*

GOMES, A. de C. et al. Composição e atividade de mosquitos Culicidae. Emprego de armadilha CDC no Vale do Ribeira, Estado de São Paulo, Brasil. Rev. Saúde públ., S. Paulo, 21:363-370, 1987.

RESUMO: Com o emprego da armadilha CDC determinou-se a composição específica da fauna de mosquitos Culicidae em locais com vegetação primária, situados em terras baixas e próximas a mangues, no litoral sul do Estado de São Paulo (Brasil). Como resultado geral foram obtidos cerca de 64 espécies ou grupos. Entre as que foram mais freqüentes destacaram-se Culex ( $\mathrm{Mel}$.) sacchettae (71,6\%), Anopheles (Ker.) cruzii $(10,5 \%)$, Phoniomiya spp. $(8,2 \%)$ e Culex (Mel.) taeniopus $(2,9 \%)$. Também com este método de coleta, nos horários crepuscular vespertino, noturno, ao lado do uso de isca ave e roedor, a dominância nítida foi de $C x$. sacchettae, ao contrário do que ocorreu no mesmo local com isca humana, quando $A n$. cruzii foi a espécie predominante. A diversidade calculada para o interior, margem da mata, campo aberto e domicílio, tendo sido de 1,7 a 6,2 revelou fauna relativamente rica para Culicidae. Não obstante a marcante presença de Culex (Cux.) quinquefasciatus no domicílio, foi inesperada a elevada frequiência de $C x$. sacchettae. Além disso, a presença proporcional de $A n$. cruzii no solo e copa sugere mobilidade desse anofelino no interior da floresta, enquanto que a sua atividade em ambiente extraflorestal revelou ser restrita. Nesse particular, $C x$. sacchettae demonstrou ser espécie ubiquiista e ocorrente durante todos os meses do ano, porém com picos mais elevados nas estações verão e outono.

UNITERMOS: Mosquitos. Armadilha CDC. Ecologia de vetores. Anopheles cruzii. Culex sacchettae.

\section{INTRODUÇÃO}

Os estudos sobre a composição e hábito da fauna culicídea adulta pertencente à floresta perenifólia higrófila, do litoral sul do Estado de São Paulo, praticamente estão delineados pelos métodos de isca humana e armadilha de Shannon (Forattini e col., ${ }^{5,6}, 1981,1986$ ).

Não obstante o valor epidemiológico dessas observações, a busca de novas técnicas de coleta constitui meta importante na investigação ecológica. Nesse particular, a armadilha CDC vem sendo amplamente aplicada com sucesso em vários países (Service $\left.{ }^{10}, 1976\right)$.

Objetivando-se ampliar o conhecimento regional sobre culicídeos e suas implicações epidemiológicas, optou-se pelo emprego desse tipo de armadilha para focalizar esse assunto. Fundamentalmente pretende-se constatar o valor da técnica no estudo da fauna geral ressaltan- do-se o hábito de Anopheles (Ker.) cruzii e Culex (Mel.) sacchettae, à luz de outros resultados obtidos simultaneamente para o mesmo local, através de metodologia distinta (Forattini e col. $\left.{ }^{6}, 1986\right)$.

Além disso, introduz-se novas informações a respeito da estratificação vertical e horizontal desses mosquitos em mata primitiva de baixada, situada junto ao ambiente desmatado.

\section{CARACTERISTICAS LOCAIS}

As atividades de coleta foram realizadas no Sítio Vilarinho. Este situa-se no Município de Cananéia, às margens da estrada que liga Itapitangui ao Ariri, a aproximadamente $3 \mathrm{~km}$ daquela Vila. Encontra-se a $24^{\circ} 57^{\prime}$ de latitude sul e $47^{\circ} 59^{\prime}$ de longitude oeste.

A região é composta de uma área de mata e outra aberta contendo uma habitação e ane-

* Departamento de Epidemiologia da Faculdade de Saúde Pública da Universidade de São Paulo - Av. Dr. Arnaldo, 715 - 01255 - São Paulo, SP - Brasil. 
xos. Sua mata é extensa, com indivíduos arbóreos de altura média de $10 \mathrm{~m}$, estando localizada na planície costeira, próximo ao mangue. Trata-se de formação primitiva, muito rica em plantas da família Bromeliaceae. Estas, com frequiência, cobrem extensas áreas do solo, sendo também comuns no estrato arbóreo. $\mathrm{Na}$ classificação de Camargo e col. ${ }^{1}$ (1972), enquadra-se como "mata tropical primária dos níveis mais baixos", sendo considerada como de "transição" pela sua contigüidade ao mangue (Forattini e col. ${ }^{6}, 1986$ ). A área aberta surgiu em parte como consequiência da extração de cascalho para restauração de estradas e em parte pela ocupação do espaço pelo homem. A Figura mostra aspectos particulares da área em estudo. A área pesquisada encontra-se também bem caracterizada por Forattini e $\mathrm{col}^{3}$ (1978).

\section{MATERIAL E MÉTODOS}

A coleta de campo foi conduzida entre março de 1983 a janeiro de 1984.

Como método de captura foi utilizada exclusivamente a armadilha CDC, modificada com proteção contra chuvas (Gomes e col. ${ }^{8}$, 1985).

Foram empregadas armadilhas sem isca animal, as quais situaram-se no intra e peridomicílio e nos estratos solo e copa do interior da mata. Armadilhas iscadas com pombo ( $\mathrm{Co}$ lumba livia) e roedor (Rattus norvegicus) se alternavam e corresponderam a pontos de coletas aos níveis do solo e da copa no interior e margem da mata, enquanto que no aberto foram instalados somente ao nível do solo. As iscas eram acopladas ao lado da armadilha, da maneira adotada por Natal $^{9}$ (1986), em pesquisa em área de matas residuais na mesma região.

Para cada armadilha iscada com animal no ambiente da mata, fazia-se coletas simultâneas sem a utilização dessa isca. Sistematizou-se o experimento em horário noturno de forma que sempre no primeiro dia de coleta utilizava-se o pombo e, no segundo, o roedor. Nesse mesmo ambiente, em coletas diurnas adotou-se o mesmo critério, porém, armadilhas sem isca animal foram excluídas.

A Tabela 1 mostra o ambiente em que cada armadilha foi instalada, o estímulo atrativo animal utilizado, o horário de coleta e a frequiência mensal das capturas. A Figura mostra a distribuição espacial dos pontos de coleta.

Todas as armadilhas instaladas ao nivel do solo obedeceram a altura de $1,5 \mathrm{~m}$, do mesmo. As alturas em relação ao solo, das armadilhas instaladas ao nível da copa, foram de $11 \mathrm{~m}$ para o interior da mata e $14 \mathrm{~m}$ para a margem.
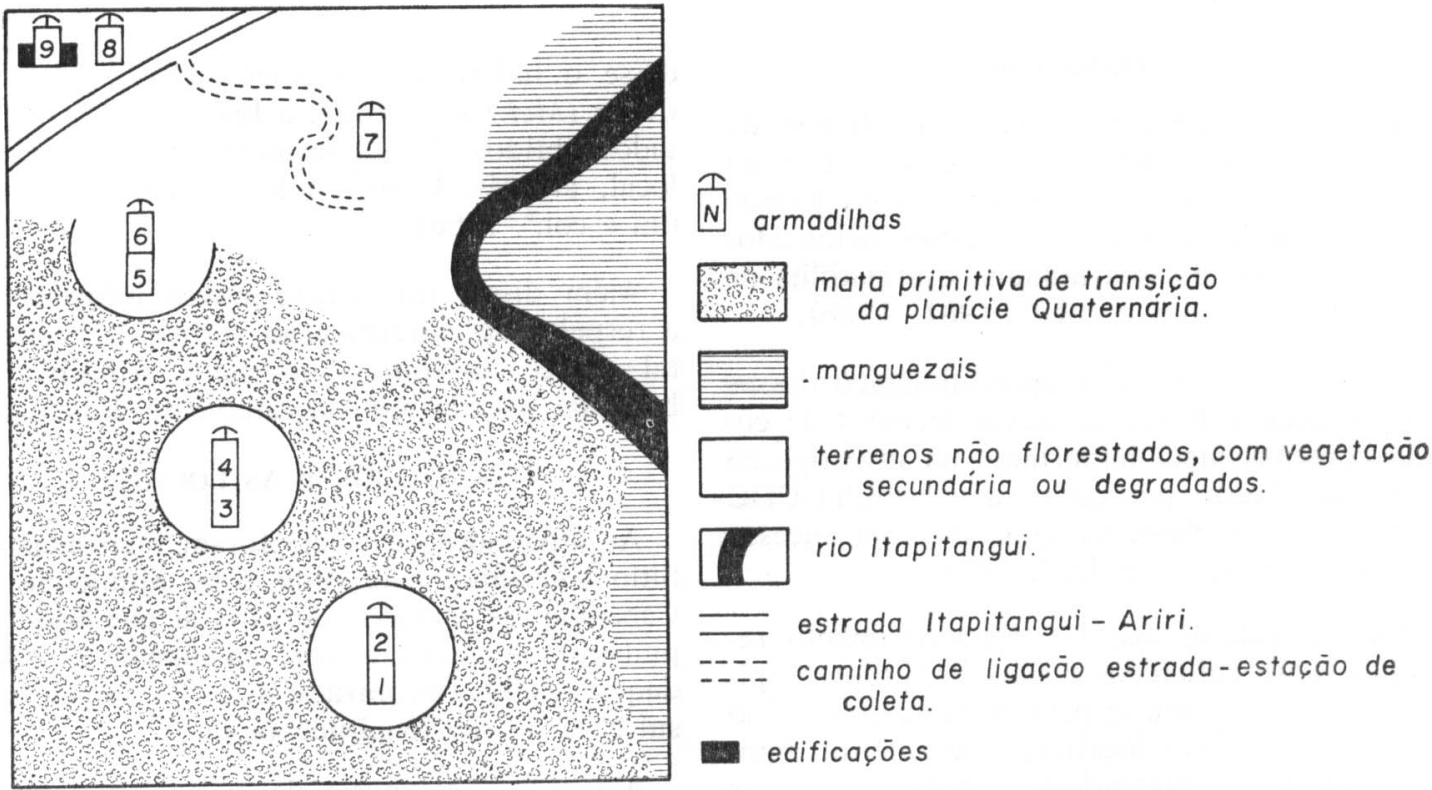

Fig. - Distribuição das armadilhas na área estudada no Sítio Vilarinho, Município de Cananéia, São Paulo: 1 - mata-solo sem isca animal; 2 - mata-copa sem isca animal; 3 - mata-solo com isca animal; 4 - mata-copa com isca animal; 5 - mata-margem-solo com isca animal; 6 - mata-margem-copa com isca animal; 7 - aberto-solo com isca animal; 8 - peridomicílio sem isca animal e 9 - intradomicílio sem isca animal. (Fonte: Forattini e col.7, 1986, com modificações). 
TABELA 1

Situação de coleta das armadilhas luminosas tipo CDC instaladas na Estação Vilarinho, Município de Cananéia, Estado de São Paulo, no período de março de 1983 a janeiro de 1984.

\begin{tabular}{|c|c|c|c|}
\hline Ambiente & $\begin{array}{c}\text { Estímulo atrativo } \\
\text { animal }\end{array}$ & Ritmo horário & $\begin{array}{l}\text { Frequiuência mensal } \\
\text { das capturas }\end{array}$ \\
\hline $\begin{array}{l}\text { Mata-solo } \\
\text { Mata-solo } \\
\text { Mata-copa } \\
\text { Mata-copa } \\
\text { Mata-solo } \\
\text { Mata-solo } \\
\text { Mata-solo } \\
\text { Mata-copa } \\
\text { Mata-copa } \\
\text { Mata-copa } \\
\text { Mata-margem-solo } \\
\text { Mata-margem-solo } \\
\text { Mata-margem-copa } \\
\text { Mata-margem-copa } \\
\text { Aberto } \\
\text { Aberto } \\
\text { Peridomicílio } \\
\text { Intradomicílio }\end{array}$ & $\begin{array}{l}\text { Pombo } \\
\text { Rato } \\
\text { Pombo } \\
\text { Rato } \\
\text { Pombo } \\
\text { Rato } \\
\text { Pombo } \\
\text { Rato } \\
\text { Pombo } \\
\text { Rato } \\
\text { Pombo } \\
\text { Rato } \\
\text { Pombo } \\
\text { Rato } \\
\text { - } \\
\end{array}$ & $\begin{array}{r}8: 00-18: 00 \\
8: 00-18: 00 \\
8: 00-18: 00 \\
8: 00-18: 00 \\
18: 00-24: 00 \\
18: 00-24: 00 \\
18: 00-24: 00 \\
18: 00-24: 00 \\
18: 00-24: 00 \\
18: 00-24: 00 \\
18: 00-24: 00 \\
18: 00-24: 00 \\
18: 00-24: 00 \\
18: 00-24: 00 \\
18: 00-24: 00 \\
18: 00-24: 00 \\
18: 00-6: 00 \\
18: 00-6: 00\end{array}$ & $\begin{array}{ll}1 & \mathrm{p} / \mathrm{mês} \\
1 & \mathrm{p} / \mathrm{mês} \\
1 & \mathrm{p} / \mathrm{mês} \\
1 & \mathrm{p} / \mathrm{mês} \\
2 & \mathrm{p} / \mathrm{mês} \\
1 & \mathrm{p} / \mathrm{mês} \\
1 & \mathrm{p} / \mathrm{mês} \\
2 & \mathrm{p} / \mathrm{mês} \\
1 & \mathrm{p} / \mathrm{mês} \\
1 & \mathrm{p} / \mathrm{mês} \\
1 & \mathrm{p} / \mathrm{mês} \\
1 & \mathrm{p} / \mathrm{mês} \\
1 & \mathrm{p} / \mathrm{mês} \\
1 & \mathrm{p} / \mathrm{mês} \\
1 & \mathrm{p} / \mathrm{mês} \\
1 & \mathrm{p} / \mathrm{mês} \\
2 & \mathrm{p} / \mathrm{mês} \\
2 & \mathrm{p} / \mathrm{mês}\end{array}$ \\
\hline
\end{tabular}

As distâncias dos pontos de coleta do interior da mata para o início do ambiente aberto foram de 60 e $115 \mathrm{~m}$, respectivamente para armadilhas iscadas com animal e não iscadas.

As distâncias em relação à mata mais próxima das armadilhas instaladas no ambiente aberto foram: em campo aberto $46 \mathrm{~m}$; no peridomicílio $70 \mathrm{~m}$ e no intradomicílio $75 \mathrm{~m}$.

Para análise geral dos dados, as espécies foram divididas em mais frequientes e menos frequientes, segundo critério adotado por Forattini e $\operatorname{col}^{5}$ (1981).

Para avaliação da diversidade específica, aplicou-se o índice de diversidade de Fisher e col. (citados por Service ${ }^{10}, 1976$ ).

Em relação à distribuição de espécies predominantes, trabalhou-se com valores percentuais.

\section{RESULTADOS}

O resultado geral, de todas as coletas, encontra-se na Tabela 2. Entre as espécies mais frequientes ocorreram Phoniomyia spp. e Culex Cx. sacchettae, com $71,6 \%$ do total coletado e An. cruzii com 10,5\%. Ainda entre as mais frequientes ocorreram Phoniomyia spp e Culex (Mel.) taeniopus com rendimentos respectivos de 8,2 e $2,9 \%$. Os demais culicídeos, representando 1.255 indivíduos, estão distribuídos em 59 espécies ou categorias superiores.

Os índices de diversidade calculados para cada ponto de coleta encontram-se na Tabela 3. Estes variam de 6,2 a 1,7.
A Tabela 4 mostra o resultado das capturas efetuadas no ambiente domiciliar. Constatou-se maior frequiência de Culex (Cux.) quinquefasciatus $(57,5 \%)$ no intradomicílio, sendo seguida de $C x$. sacchettae $(22,2 \%)$ e Anopheles (Ker.) bellator $(6,5 \%)$. No peridomicílio houve dominância de $C x$. sacchettae $(68,1 \%)$, vindo a seguir $A n$. cruzii $(11,0 \%)$.

A distribuição horizontal e vertical de $A n$. cruzii e $C x$. sacchettae pode ser vista na Tabela 5 . Observa-se, assim, a marcante presença de $A n$. cruzii na copa da margem da mata independentemente de isca utilizada. Em relação à $C x$. sacchettae, ocorreu de maneira não muito diferenciada em todos os pontos de coleta, com ligeira tendência para maior concentração no solo. Evidenciou-se maior preferência para isca rato no ambiente aberto.

A Tabela 6 revela a distribuição mensal de fêmeas de $C x$. sacchettae para a área estudada, segundo a isca animal utilizada. A análise dessa mostrou variação de densidade ao longo do ano, com maiores frequiências nos meses quentes e menores nos frios. Também foi verificada tendência de mudança na preferência em relação à isca, sendo que no início do ano armadilhas com isca pombo tiveram maiores rendimentos, ao passo que nos meses finais ocorreu uma inversão de situação, com maiores percentuais nas armadilhas iscadas com rato.

Dado que o ambiente de maior freqüência de $A n$. cruzii foi representado pela copa da margem da mata, relacionou-se as armadilhas 
TABELA 2

Distribuição de culicídeos na Estação Vilarinho, Município de Cananéia, Estado de São Paulo, no período de março de 1983 a janeiro de 1984.

\begin{tabular}{lrr}
\hline \multicolumn{1}{c}{ Espécies (mais freqüentes) } & N. & \multicolumn{1}{c}{$\%$} \\
\hline Culex (Melanoconion) sacchettae & 13.169 & 71,6 \\
Anopheles (Kerteszia) cruzii & 1.933 & 10,5 \\
Phoniomyia spp. & 1.512 & 8,2 \\
Cx. (Mel.) taeniopus & 524 & 2,9 \\
Outras espécies & $1.255(\mathrm{a})$ & 6,8 \\
$\quad$ (menos frequientes) & 18.393 & 100,0 \\
\hline \multicolumn{2}{c}{ Total } & \\
\hline
\end{tabular}

(a)* Culex (Culex)spp.; An. (Ker.)bellator; Aedes (Ochlerotatus) serratus; Ph. davisi; Coquillettidia (Rhynchotaenia) chrysonotum; Cx. (Mel.) spp.; Cx. (Mel.) ribeirensis; Ae. (Och.) scapularis; $C x$. (Cux.) nigripalpus; $C x$. (Cux.) coronator; Cx. (Mel.) lopesi; Cq. (Rhy.) venezuelensis; Cx. (Mel.) pedroi; Runchomyia (Runchomyia) reversa; Limatus durhami; CX. (Mel.) misionensis; Psorophora (Janthinosoma) ferox; Wyeomyia (Dendromyia) confusa; $C x$. (Mel.) spp. grupo pilosus; Cx. (Cux.) declarator; Ru. (Run.) theobaldi; Ru. (Run.) frontosa; Cx. (Cux.) mollis; Cx. (Cux.) dolosus; Wy. (Den.) aporonoma; $C x$. (Mel.) corentynensis; Wy. (Den.) sp. próx. knabi; Runchomyia spp.; Cx. (Microculex) imitador; Cx. (Cux.) lygrus; Wy. (Den.) sp. próx. aporonoma; Wy. (Den.) sp. próx. mystes; Sabethes (Sabethes) purpureus; Cq. (Rhy.) juxtamansonia; An. (Anopheles) intermedius; Ae. (Och.) crinifer; Wyeomyia spp.; Wy. (Den.) sp. próx. airosai; $C x$. (Mcx.) neglectus; Cx. (Mel.) spp. grupo atratus; Cx. (Mel.) theobaldi; An. (Ano.) mediopunctatus; Uranotaenia (Uranotaenia) lowii; Sa. (Sabethinus) undosus; Sa. (Sab.) shannoni; Sa. (Sab.) quasicyaneus; Ps. (Grabhamia) cingulata; Li. flavisetosus; $C x$. (Mel.) pereyrai; $C x$. (Cux.) bidens; Ur. (Ura.) pallidoventer; Trichoprosopon (Trc.) pallidiventer; Sa. (Sbn.) intermedius; Ps. (Gra.) confinnis; Ps. (Jan.) albigenu; Mansonia (Mansonia) spp.; $C x$. (Mel.) inadmirabilis; Cx. (Mel.) evansae.

* Em ordem decrescente de freqüência.

desse ponto para o estudo da distribuição mensal dessa espécie. Os dados encontram-se na Tabela 7 . Os resultados obtidos foram semelhantes àqueles de $C x$. sacchettae, ocorrendo também ao longo do ano a inversão de preferência pelas diferentes iscas.

\section{COMENTÁRIOS}

As observações sobre a composição específica de mosquitos Culicidae levadas a cabo em matas de terras baixas e próximas a manguezais, através da armadilha $\mathrm{CDC}$, revelaram a existência de 64 espécies ou grupos, entre as quais se destacam a marcante presença de $C x$. sacchettae $(71,6 \%)$, seguida de $10,5 \%$ para
An. cruzii (Tabela 2). Em coletas simultâneas com isca humana e armadilhas de Shannon, na mesma localidade, foi constatado rendimento diferenciado entre as três técnicas empregadas. Desse modo, com isca humana a distribuição proporcional foi de $25,9 \%$ para An. cruzii, $16,4 \%$ para $C x$. sacchettae e $48,2 \%$ para o gênero Phoniomyia (Forattini e col. ${ }^{7}$, 1986). Nesse mesmo relato os autores mostraram que, com armadilha de Shannon, $A n$. cruzii predominou com $70,7 \%$ e $C x$. sacchettae com $15,7 \%$. Ora, levando-se em conta que o horário de captura para as armadilhas CDC e Shannon foram iguais e que somente o período para isca humana foi de 25 horas ininterruptas, comprova-se que houve diferença de atração populacional específica entre as técnicas usadas para a área estudada. Fato também adverso em relação aos resultados obtidos em matas residuais com armadilha $\mathrm{CDC}$, com e sem isca animal (Gallus gallus), visto que a frequiência de $C x$. sacchettae fora apenas de $11,2 \%$ (Natal $\left.{ }^{9}, 1986\right)$. Portanto, as variações percentuais assinaladas nos locais estudados indicaram métodos satisfatórios para a identificação de ambientes mais preferidos por $C x$. sacchettae. Deduz-se ainda desta análise comparativa o conhecimento de que a medida da dominância local entre os mosquitos, numa biocenose ou ecossistema, sofre a influência do aspecto seletivo que cada técnica de coleta encerra. Apesar disto, há de se reconhecer a importância epidemiológica da isca humana.

A diversidade aqui encontrada de 64 espécies ou grupos de categorias indicou índices de Fischer e col. (citados por Service ${ }^{10}, 1976$ ) que variaram nos diversos pontos de coleta, de 1,7 a 6,2 , o que mostra fauna relativamente rica. Esta situação, quando comparada à área alterada, foi menor pois o mesmo índice variou de 6,3 a 12,0 para 118 espécies ou grupo $\left(\mathrm{Natal}^{9}, 1986\right)$. Isto sugere que se métodos de coleta menos seletivos fossem empregados, seria possível encontrar maior diversidade nesse tipo particular de ambiente.

Várias espécies (Tabela 4) foram coletadas no ambiente domiciliar. A elevada freqüência de $C x$. quinquefasciatus no domicílio não se constituiu uma novidade, dada a sua reconhecida adaptação a este ecótopo. Todavia, chama atenção $C x$. sacchettae que apareceu em número expressivamente maior no domicílio do que em peridomicílio. An. cruzii com 2,8 e $11,0 \%$, respectivamente nos mesmos locais, trata-se de espécie bastante comum e agressiva ao homem, na área. Assim, Forattini e col. ${ }^{6}$ (1986) fazem referência a este aspecto, assi- 
TABELA 3

Diversidade de culicídeos* em coletas efetuadas nos diversos pontos da Estação Vilarinho, Município de Cananéia, Estado de São Paulo, no período de março de 1983 a janeiro de 1984.

\begin{tabular}{lrlrrr}
\hline \multicolumn{1}{c}{ Local } & Horário & \multicolumn{1}{c}{ Isca } & $\begin{array}{c}\text { Número de } \\
\text { espécies }\end{array}$ & $\begin{array}{c}\text { Número de } \\
\text { indivíduos }\end{array}$ & $\begin{array}{c}\text { Indice de } \\
\text { diversidade }\end{array}$ \\
\hline Mata-solo & $8: 00-18: 00$ & Pombo & 21 & 180 & 6,2 \\
Mata-copa & $18: 00-24: 00$ & Rato & 23 & 838 & 4,4 \\
Mata-solo & $8: 00-18: 00$ & Rato & 18 & 268 & 4,3 \\
Mata-copa & $18: 00-24: 00$ & Sem animal** & 20 & 561 & 4,1 \\
Mata-solo & $18: 00-24: 00$ & Sem animal** & 20 & 703 & 3,8 \\
Aberto-solo & $18: 00-24: 00$ & Rato & 22 & 2.168 & 3,4 \\
Mata-margem-solo & $18: 00-24: 00$ & Pombo & 22 & 2.200 & 3,4 \\
Peridomicílio & $18: 00-6: 00$ & Sem animal** & 16 & 364 & 3,4 \\
Mata-copa & $8: 00-18: 00$ & Pombo & 16 & 451 & 3,2 \\
Mata-solo & $18: 00-24: 00$ & Rato & 19 & 1.516 & 3,1 \\
Mata-copa & $18: 00-24: 00$ & Pombo & 19 & 1.339 & 3,1 \\
Intradomicílio & $18: 00-6: 00$ & Sem animal** & 10 & 77 & 3,1 \\
Mata-margem-solo & $18: 00-24: 00$ & Rato & 19 & 1.902 & 2,9 \\
Mata-margem-copa & $18: 00-24: 00$ & Pombo & 17 & 1.454 & 2,7 \\
Mata-solo & $18: 00-24: 00$ & Pombo & 17 & 1.572 & 2,7 \\
Mata-copa & $8: 00-18: 00$ & Rato & 15 & 1.011 & 2,5 \\
Aberto-solo & $18: 00-24: 00$ & Pombo & 13 & 1.228 & 2,0 \\
Mata-margem-copa & $18: 00-24: 00$ & Rato & 11 & 965 & 1,7 \\
\hline
\end{tabular}

* Somente as fêmeas foram consideradas.

** Duas coletas por mês.

\section{TABELA 4}

Distribuição de culicídeos coletados no ambiente domiciliar da Estação Vilarinho, Município de Cananéia, Estado de São Paulo, no período de março de 1983 a janeiro de 1984.

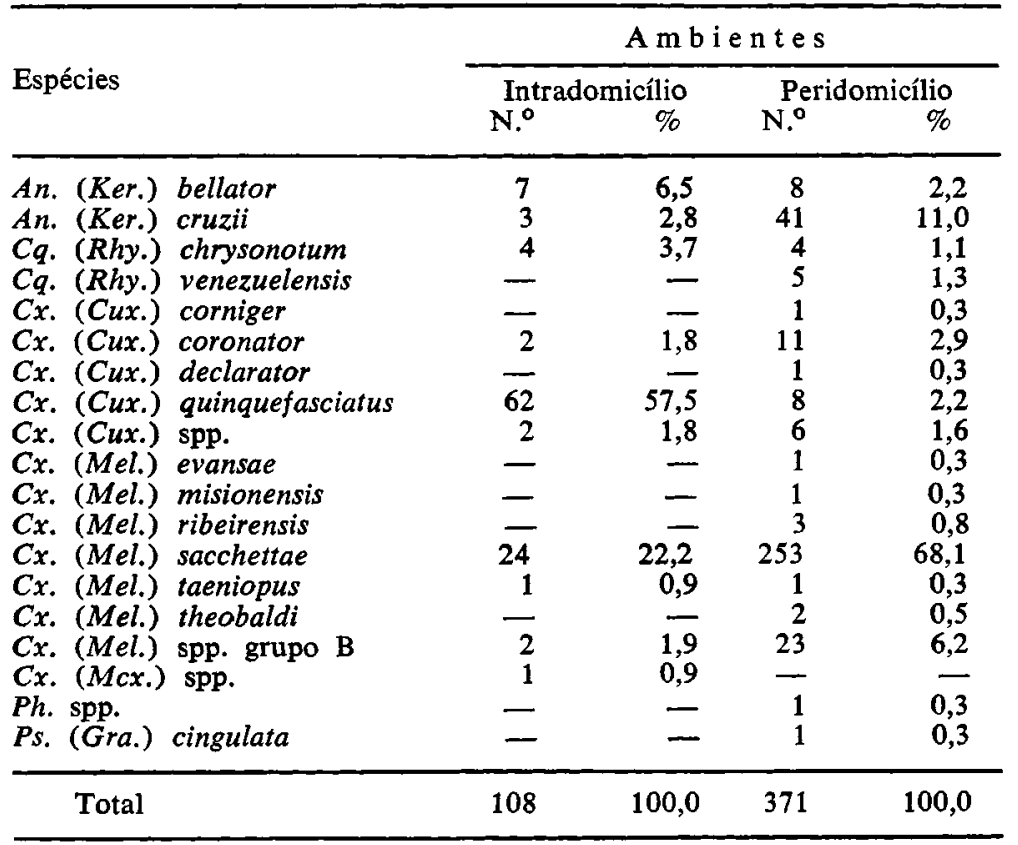

nalando a proporção de $34,1 \%$ em coleta com isca humana e armadilha de Shannon. Já na estação Folha Larga, próximo ao Sítio Vilarinho, a presença desta espécie foi de $46,9 \%$. An. bellator embora possa manifestar certo grau de endofagia, os resultados não podem ser ainda conclusivos. Além disso, tem-se verificado a significativa presença de Anopheles através de técnica de coleta que empregam o homem como isca, fato este que evidencia também o comportamento antropófilo de $A n$. cruzii. Portanto, tais conhecimentos revelam 
TABELA 5

Distribuição horizontal e vertical de fêmeas de espécies predominantes no horário noturno, segundo a isca utilizada, na Estação Vilarinho, Município de Cananéia, Estado de São Paulo, no período de março de 1983 a janeiro de 1984.

\begin{tabular}{|c|c|c|c|c|c|c|c|c|}
\hline Espécies & \multicolumn{4}{|c|}{ An. cruzii } & \multicolumn{4}{|c|}{ Cx. sacchettae } \\
\hline Isca & \multicolumn{2}{|c|}{ Pombo } & \multicolumn{2}{|c|}{ Rato } & \multicolumn{2}{|c|}{ Pombo } & \multicolumn{2}{|c|}{ Rato } \\
\hline Ambientes & $\mathrm{N}^{\circ}$ & $\%$ & N. ${ }^{\circ}$ & $\%$ & N. ${ }^{\circ}$ & $\%$ & N. ${ }^{\circ}$ & $\%$ \\
\hline $\begin{array}{l}\text { Mata-solo } \\
\text { Mata-copa } \\
\text { Mata-margem-solo } \\
\text { Mata-margem-copa } \\
\text { Aberto }\end{array}$ & $\begin{array}{r}15 \\
72 \\
193 \\
598 \\
29\end{array}$ & $\begin{array}{r}1,7 \\
7,9 \\
21,3 \\
65,9 \\
3,2\end{array}$ & $\begin{array}{r}34 \\
193 \\
105 \\
548 \\
86\end{array}$ & $\begin{array}{r}3,5 \\
20,0 \\
10,9 \\
56,7 \\
8,9\end{array}$ & $\begin{array}{r}1.409 \\
1.164 \\
1.834 \\
750 \\
1.094\end{array}$ & $\begin{array}{l}22,5 \\
18,6 \\
29,4 \\
12,0 \\
17,5\end{array}$ & $\begin{array}{r}1.186 \\
468 \\
1.591 \\
345 \\
1.921\end{array}$ & $\begin{array}{r}21,5 \\
8,5 \\
28,8 \\
6,3 \\
34,9\end{array}$ \\
\hline Total & 907 & 100,0 & 966 & 100,0 & 6.251 & 100,0 & 5.511 & 100,0 \\
\hline
\end{tabular}

\section{TABELA 6}

Distribuição mensal de fêmeas de $C x$. sacchettae, segundo a isca animal utilizada em coletas noturnas, na Estação Vilarinho, Município de Cananéia, Estado de São Paulo, no período de março de 1983 a janeiro de 1984.

\begin{tabular}{|c|c|c|c|c|c|}
\hline \multirow[b]{2}{*}{ Meser/anos } & \multirow[t]{2}{*}{ Iscas } & \multicolumn{2}{|c|}{ Pombo* } & \multicolumn{2}{|c|}{ Rato* } \\
\hline & & N.o & $\%$ & N. ${ }^{\circ}$ & $\%$ \\
\hline Março/83 & & 319 & 5,1 & 1.041 & 18,9 \\
\hline Abril/ 83 & & 1.023 & 16,4 & 400 & 7,3 \\
\hline Maio/83 & & 1.699 & 27,2 & 481 & 8,6 \\
\hline Junho/83 & & 81 & 1,3 & 30 & 0,5 \\
\hline Julho/83 & & 6 & 0.1 & - & - \\
\hline Agosto/83 & & 179 & 2,9 & 20 & 0,4 \\
\hline Setembro/83 & & 520 & 8,3 & 528 & 9,6 \\
\hline Outubro/83 & & 1.095 & 17,5 & 219 & 4,0 \\
\hline Novembro/83 & & 778 & 12,4 & 958 & 17,4 \\
\hline Dezembro/83 & & 519 & 8,3 & 1.764 & 32,0 \\
\hline Janeiro/84 & & 32 & 0,5 & 70 & 1,3 \\
\hline Total & & 6.251 & 100,0 & 5.511 & 100,0 \\
\hline
\end{tabular}

* Armadilhas instaladas com as referidas iscas nos ambientes da mata e margem da mata aos níveis do solo e da copa e na área aberta.

\section{TABELA 7}

Distribuição mensal de fêmeas de $A n$. cruzii segundo a isca animal utilizada, no ambiente da copa da margem da mata, em coletas noturnas, na Estação Vilarinho, Municipio de Cananéia, Estado de São Paulo, no periodo de março de 1983 a janeiro de 1984.

\begin{tabular}{|c|c|c|c|c|}
\hline \multirow{2}{*}{$\begin{array}{l}\text { Iscas } \\
\text { Meses/anos }\end{array}$} & \multicolumn{2}{|c|}{ Pombo } & \multicolumn{2}{|c|}{ Rato } \\
\hline & N. ${ }^{\circ}$ & $\%$ & N. ${ }^{\circ}$ & $\%$ \\
\hline Março/83 & 111 & 18,6 & 5 & 0,9 \\
\hline Abril/ 83 & 353 & 59,0 & 1 & 0,2 \\
\hline Maio/83 & 73 & 12,2 & 40 & 7,3 \\
\hline Junho $/ 83$ & 2 & 0,3 & 3 & 0,5 \\
\hline Julho/83 & - & - & - & - \\
\hline Agosto/83 & 1 & 0,2 & 29 & 5,3 \\
\hline Setembro/83 & 11 & 1,8 & - & - \\
\hline Outubro/83 & 27 & 4,5 & 1 & 0,2 \\
\hline Novembro/83 & 1 & 0,2 & 192 & 35,0 \\
\hline Dezembro/83 & 19 & 3,2 & 270 & 49,3 \\
\hline Janeiro/84 & - & - & 7 & 1,3 \\
\hline Total & 598 & 100,0 & 548 & 100,0 \\
\hline
\end{tabular}


condição potencial para a ocorrência de transmissão domiciliar da malária na região estudada, sobretudo pela antecedente comprovação de endofagia deste anofelino na região do Vale do Ribeira (Forattini e col. ${ }^{4}, 1978$ ).

O fato de $A n$. cruzii apresentar comportamento acrodendrófilo já era esperado, uma vez que esta espécie tem seus criadouros em bromélias epífitas. Quanto ao seu comparecimento nos aparelhos $\mathrm{CDC}$, em número não muito elevado, mas constante e proporcional entre o nível da copa e solo, conduz à suposição que a atividade hematófaga se faça obedecendo mobilidade entre os estratos da floresta e em direção também ao ambiente extraflorestal incluindo as habitações próximas. A feição florestal desta espécie vai ao encontro do relato de Deane e col. ${ }^{2}$ (1984). Acresce ainda que, mesmo levando-se em conta as iscas pombo e rato, apesar dos resultados não serem uniformes, persiste 0 consenso que $A n$. cruzii e $C x$. sacchettae tenham comportamento similar na área (Tabela 5), porém o segundo parece possuir capacidade de invasão do ambiente aberto, discretamente superior ao primeiro. Por outro lado, não foi confirmado aqui a preferência de $C x$. sacchettae pelo ecótono conforme já havia sido anteriormente observado por $\mathrm{Natal}^{9}$ (1986).

Com relação à capacidade dispersa das espécies em questão parece patente que $C x$. sacchettae se caracteriza como população ubiquiista, pois foi assinalada da mata ao intradomicídio, cuja proporção no ambiente aberto corresponde a $34,9 \%$. Corrobora com isto as observaçōes de Forattini e col. ${ }^{6}$ (1986) que, com armadilha de Shannon, mostraram ser a mais frequiente também naquele local. Todavia, com base no perfil populacional por ambiente, determinado na mesma investigação, sugere-se que em ambiente com elevado grau de alteração, esta espécie venha ser sucedida na dominância por Culex (Mel.) ribeirensis.

Os resultados das coletas mensais evidenciaram a presença de $C x$. sacchettae praticamente durante todos os meses do ano, entretanto os picos de atividade detectados variaram em função da isca animal empregada (Tabela 6). Assim, as coletas com isca pombo renderam mais em abril, maio e outubro, enquanto que com isca rato corresponderam a março e dezembro. Não obstante a ausência de dados sobre a participação de chuvas na área estudada, deduz-se que em termos estacionais o verão e outono seriam épocas de maior reprodução deste mosquito. No que concerne a $A n$. cruzii, confirmam-se as observaçōes de Forattini e col. ${ }^{6}$ (1986), quando nas duas situações ficou evidenciado que as estaçōes frias (inverno-outono) corresponderiam a períodos de maior redução da atividade dos adultos deste mosquito. Além disso, a influência das iscas animais empregadas para atrair maior número de indivíduos transpareceu ter sido discreta quando comparada a $C x$. sacchettae (Tabela 7). Portanto, no presente trabalho, este tipo de comportamento pressupõe que para as duas espécies ocorreu uma aparente mudança de preferência hospedeira, porém os dados não não foram considerados suficientes para se concluir por hábito eclético, sem que dispusesse da identificação do conteúdo alimentar, através da reação de precipitina.

\section{CONCLUSO̊ES}

Coletas com armadilha CDC sem isca animal ou associada à isca pombo e rato, levadas a efeito em floresta de transição e situada entre o mangue e mata de encosta, indicaram que:

1. Como técnica de coleta a referida armadilha mostrou resultados satisfatórios à análise do comportamento de $C x$. sacchettae e An. cruzii.

2. A medida de dominância local sofreu influência de cada técnica de coleta utilizada, porém ressaltando-se que com CDC, $C x$. sacchettae foi a que mais se destacou.

3. Resguardadas as distâncias relativamente pequenas entre a habitação humana e ponto de coleta do aberto, julga-se pela densidade que $C x$. sacchettae possua característica ubiqüista, o mesmo não sendo possível ser atribuído a $A n$. cruzii.

4. A atividade de $C x$. sacchettae ocorreu durante todo o ano, mas as estações verão $e$ outono foram as que apresentaram picos mais expressivos, inclusive sendo esses influenciados pela isca animal.

5. A análise sobre $C x$. sacchettae sugere preferência pelo ecótono ao nível do solo, enquanto que $A n$. cruzii mostrou-se indiferente aos estratos inferiores e superiores da floresta. 
GOMES. A. de C. et al. [Determination of the composition and activity of Culicidae mosquitoes by the use of the CDC trap in the Ribeira Valley, S. Paulo State, Brazil]. Rev. Saúde públ., S. Paulo, 21:363-370, 1987.

ABSTRACT: Using a CDC light-trap, the specific fauna composition of mosquitoes Culicidae was determined at places with primary vegetation of lowlands near swampy areas along the Southern coast of S. Paulo State, Brazil. As a result, about 64 species or groups were found. Among the more frequent ones, Culex ( $\mathrm{Mel}$.) sacchettae (71.6\%), Anopheles (Ker.) cruzii (10.5\%), Phoniomiya spp. (8.2\%) and Culex (Mel.) taeniopus $(2.9 \%)$ were outstanding. Also by the use of this method of capture, with fowls and rodents as animal bait, in vespertine dusk and night periods, the $C x$. sacchettae showed a clear dominance, which was the opposite of what occured at the same places, using human bait, when $A n$. cruzii was the predominant species. Ranging from 1.7 to 6.2 , the diversity estimated for inland, edge forest, open field and residential areas showed a relatively rich fauna of Culicidae. Despite the marked presence of Culex (Cux.) quinquefasciatus in domiciliary area, the high frequence of $C x$. sacchettae was rather unexpected. Moreover, the proportional presence of $A n$. cruzii, both on the ground and in forest canopy suggests the mobility of this anophelinae inside the wood, while its activity in extraforest environments has revealed itself to be very restricted. In this particular case, $C x$. sacchettae has shown itself as the most ubiquitons and frequently occurring species throughout the year, although its higher density peaks occur in summer and autumn.

UNITERMS: Mosquitoes. CDC trap. Ecology, vectors. Anopheles cruzii. Culex sacchettae.

\section{REFERENCIAS BIBLIOGRÁFICAS}

1. CAMARGO, J. C. G. et al. Estudos fitogeográficos e ecológicos da bacia hidrográfica paulista do Rio Ribeira. São Paulo, Instituto de Geografia da USP, 1972. (Série Biogeografia, 5).

2. DEANE, L. M. et al. The vertical dispersion of Anopheles (Kerteszia) cruzii in a forest in southern Brazil suggests that human case of malaria of simian origin might be expected. Mem. Inst. Oswaldo Cruz, Rio de Janeiro, 79:461-3, 1984.

3. FORATTINI, O. P. et al. Estudos ecológicos sobre mosquitos Culicidae no sistema da Serra do Mar, Brasil. 1 - Observaçóes no ambiente extradomiciliar. Rev. Saúde públ., S. Paulo, 12:297-325, 1978.

4. FORATTINI, O. P. et al. Estudos ecológicos sobre mosquitos Culicidae no sistema da Serra do Mar, Brasil. 2 - Observações no ambiente domiciliar. Rev. Saúde públ., S. Paulo, 12:476-96, 1978.

5. FORATTINI, O. P. et al. Observações sobre atividades dos mosquitos Culicidae, em mata residual no Vale do Ribeira, São Paulo, Brasil. Rev. Saúde públ., S. Paulo, 15:557-86, 1981.
6. FORATTINI, O. P. et al. Ubservaçōes sobre atividade de mosquitos Culicidae em mata primitiva da encosta no Vale do Ribeira, São Paulo, Brasil. Rev. Saúde públ., S. Paulo, 20: $1-20,1986$

7. FORATTINI, O. P. et w1. Observações sobre atividade de mosquitos Culicidae em matas primitivas de planície e perfis epidemiológicos de vários ambientes no Vale do Ribeira, São Paulo, Brasil. Rev. Saúde públ., S. Paulo, 20:178-203, 1986.

8. GOMES, A. de C. eı al. Uma nova câmara coletora para armadilha CDC-miniatura. Rev. Saúde públ., S. Paulo, 19:190-1, 1985.

9. NATAL, D. Observaçōes sobre o comportamento de mosquitos (Diptera: Culicidae) em área de matas residuais no Vale do Ribeira, Estado de São Paulo, Brasil. São Paulo, 1986. [Tese de Doutorado - Faculdade de Saúde Pública da USP].

10. SERVICE, M. W. Mosquito ecology-field sampling methods. London, Applies Science Publishers, 1976.

Recebido para publicação em: 26/6/1987 A provado para publicação em: 10/8/1987 